\title{
Optical Engineering
}

SPIEDigitalLibrary.org/oe

\section{Errata: Spectrum reconstruction for filter-array spectrum sensor from sparse template selection}

Cheng-Chun Chang

Nan-Ting Lin

Umpei Kurokawa

Choi Byung II 


\title{
Errata: Spectrum reconstruction for filter-array spectrum sensor from sparse template selection
}

\author{
Cheng-Chun Chang \\ Nan-Ting Lin \\ National Taipei University of Technology \\ Department of Electrical Engineering \\ Taipei 106, Taiwan \\ E-mail: ccchang@ntut.edu.tw \\ Umpei Kurokawa \\ Choi Byung II \\ Nanolambda Incorporated \\ 510 William Pitt Way \\ Pittsburgh, Pennsylvania 15238 \\ [DOI: 10.1117/1.OE.51.5.059804]
}

This article [Opt. Eng. 50, 114402 (2012)] was originally published on 25 Oct. 2011 with an error in the Acknowledgments. The corrected statement appears below:

"This work was supported in part by the National Science Council of R.O.C. under Grant No. 982218-E-027-016-MY3." 